\title{
Neuronal mechanisms of learning in teleost fish
}

\section{Mecanismos neuronales del aprendizaje en peces teleósteos}

Recibido: marzo 10 de 2010 | Revisado: abril 21 de 2010 | Aceptado: abril 25 de 2010

\author{
CAmilo Hurtado-ParRado ** \\ University of Manitoba, Winnipeg, Canada
}

Para citar este artículo. Hurtado-Parrado, C. (2010) Neuronal mechanisms of learning in teleost fish. Universitas Psychologica, 9 (3), 657-672.

* This article was partly supported by a scholarship from the Natural Sciences and Engineering Research Council of Canada (NSERC).

The author wishes to thank Kate Dubberley for her help during the preparation of this paper, and to Drs. Randall Jamieson, James Hare, Les Leventhal, and Joseph Pear for their comments on a previous version of this document.

** Correspondence concerning this paper can be sent to 206 Chancellor's Hall, 177 Dysart Rd. University of Manitoba, Winnipeg, MB R3T 2N2-Canada Email: umhurtah@cc.umanitoba.ca

\section{A B S T R A C T}

Two predominant approaches for studying the neurobiology of learning in fish are reviewed: brain lesions and chemical stimulation. Habituation, sensitization, Pavlovian Conditioning, spatial behavior, and emotional learning are the specific processes analyzed. Regarding the effect of brain lesions, telencephalic ablations produced impairment of habituation learning; conversely, cerebellum lesions caused deficiencies in classical conditioning of eye-retraction and spatial learning (similar effects observed in mammals suggest that the functions of the cerebellum may have evolved early in vertebrate history). Medium Pallium (MP) areas have been identified as critical for emotional learning in fish. Furthermore, neurobehavioral functions of MP seem to be similar to the functions of the amygdala in mammals. Relating to neurochemical processes, NMDA receptor antagonists affected the acquisition of avoidance and fear conditioning in a dose-dependent manner. Alternatively, Nitric Oxide (NO) and cyclic Guanosine Monophosphate (cGMP) seem to be involved in the consolidation process of emotional learning.

Keywords author

Neurobehavioral functions, neurochemical functioning, teleost fish, learning processes, spatial learning, Medium Lateral Pallium.

Keywords plus

Teleost Fish, Neurochemistry, Cerebrum, Injuries.

\section{RES U MEN}

Se revisaron dos aproximaciones al estudio de la neurobiología del aprendizaje en peces teleósteos: lesiones cerebrales y estimulación química. Respecto al efecto de lesiones cerebrales, la literatura reporta que las ablaciones del telencéfalo producen deficiencias en habituación, mientras que las lesiones en el cerebelo afectan el condicionamiento clásico de retracción ocular y aprendizaje espacial (efectos similares observados en mamíferos sugieren que las funciones del cerebelo pudieron haber evolucionado tempranamente en la historia de los vertebrados). Áreas del Medium Pallium (MP) parecen ser vitales en el aprendizaje emocional de los peces; más aún, las funciones del MP aparentan ser similares a las de la amígdala en mamíferos. Con respecto a procesos neuroquímicos, los antagonistas de los receptores NMDA, mostraron afectar la adquisición de condicionamiento de evitación y miedo. Por último, el óxido nítrico y el guanosín monofosfato cíclico han sido relacionados con los procesos de consolidación del aprendizaje emocional. Palabras clave autor

Funcionamiento neuroquímico, peces teleósteos, aprendizaje espacial, procesos de aprendizaje, Medium Pallium Lateral.

Palabras clave descriptor

Peces teleósteos, neuroquímica, cerebro, lesiones. 
This paper reviews the literature specialized in the identification of the neurological mechanisms that underlie different learning processes in teleost fish. Given that one of the main interests of the reviewed research programs has been to demonstrate homologies of brain structures and behavioral functions between mammals and fish, the paper starts describing a) the basic assumptions of the neuroethological approach, followed by b) a brief description of brain anatomy and ontogeny of fish, and c) the evolutionary implications that stem from the analysis of the forebrain development of fish. The main idea of this introductory section is that, contrary to the most accepted assumption held in the comparative field until the late twentieth century, recent evidence supports the existence of homologies in brain and behavioral functions when comparing fish and mammals.

After discussing the evolutionary approach and the general hypothesis about possible homologies between fish and mammals, the paper provides details regarding the evidence that supports the relationship between different areas of the fish brain, neurochemical functioning, and several learning phenomena. Habituation, sensitization, Pavlovian conditioning, spatial behavior and emotional learning will be the specific processes reviewed. Furthermore, most of the evidence presented will be based on experiments which tested the effects of complete or partial forebrain ablations, or the effects of chemical stimulation in different parts of the fish brain. In general, strong evidence supports that the pallium areas (medium and lateral), the cerebellum, and the chemical processes involved in Long-Term Potentiation have an important role in the emergence and maintenance of learned behavior.

\section{Analysis of brain mechanisms and behavior: The neuroethological approach}

An area within the neurosciences that is specialized in the comparative study of both the neural anatomy and the functions that underlie animal behavior is termed Comparative Neuroethology (Laming, 1981). As a multidisciplinary area, Comparative Neuroethology integrates knowledge from different disciplines (e.g., evolutionary biology, neuroanatomy, ethology, physiology, and psychology) and has its own methods and sources of evidence.

This paper is almost entirely based on neuroethological experimental procedures and related evidence, therefore, some conceptual and methodological assumptions are presented here as guidelines to understand the rationale behind the conclusions and orientations of the research that was reviewed. These assumptions are the following: a) the knowledge regarding the ontogeny of the brain and comparative brain anatomy, physiology and behavior, are two of the most important sources of information to understand the evolution of the vertebrate brain mechanisms; b) the advantages of comparative studies lie in the ability to examine the functions within the brain and the associated behavior in the fully developed animal; c) the constancy of embryological brain development in vertebrates and the recapitulation of evolution that occurs during ontogeny have provided important information for understanding brain morphology; and d) the information about brain morphology is, nevertheless, less helpful when further understanding of physiology and behavior are attempted, especially due to technical and interpretative limitations.

Lastly, one of the major difficulties for the neuroethological studies is the extrapolation of results from a particular vertebrate species to others. This limitation applies especially in those cases where common ancestors ceased to exist a long time ago and today's species are not members of a linear phylogenetic scale (instead they tend to represent variations along a "tree" whose trunk does not exist anymore, Laming, 1981). Although extrapolations in general are carefully reviewed under the neuroethological approach, they still seem to be possible alternatives, especially when they relate to basic brain functions such as learning (Laming, 1981). 


\section{General characteristics of the fish brain and its ontogeny}

A common finding among vertebratesis that the nervous system is one of the earliest groups of tissues to develop embryologically. As Laming (1981) points out, the neural tube is fully developed by the time that $10-15 \%$ of embryonic life has passed. Moreover, from an evolutionary perspective, the thickening of the neural tube is evidence of the cephalization of both the sense organs and integrative centers that has occurred in vertebrates.

According to Laming (1981):

(...) Outgrows of this early developed nervous system and connections with nervous tissue outside the neural tube form the spinal and cranial nerves. These are the routes by which the animal receives information from its own tissues and the environment [; also, those structures] relay commands to muscles, glands and sense organs through which a response is mediated. (p. 9)
The neural tube develops into three components: the prosencephalon (forebrain), mesencephalon (mid-brain), and rhombencephalon (hindbrain). These three vesicles are traditionally associated with the three primary senses-olfaction, vision, and audition respectively which together, constitute the brainsteam in adult vertebrates. Further in development, each one of these vesicles develops a secondary outgrowth: telencephalon (cerebrum), optic tectum, and cerebellum respectively (Davis \& Northcutt, 1983; Laming, 1981).

A general overview of the major brain regions, divisions, locations and their respective abbreviations is displayed in Figure 1.

\section{Evolution and development of the forebrain in fish}

This paper is mainly dedicated to the description of the neuroethological research that supports the idea that certain structures of the forebrain are critical for the learning processes of one of the oldest vertebrate family. Therefore, it is necessary

FIGURE 1

Overview of a generalized lower vertebrate brain
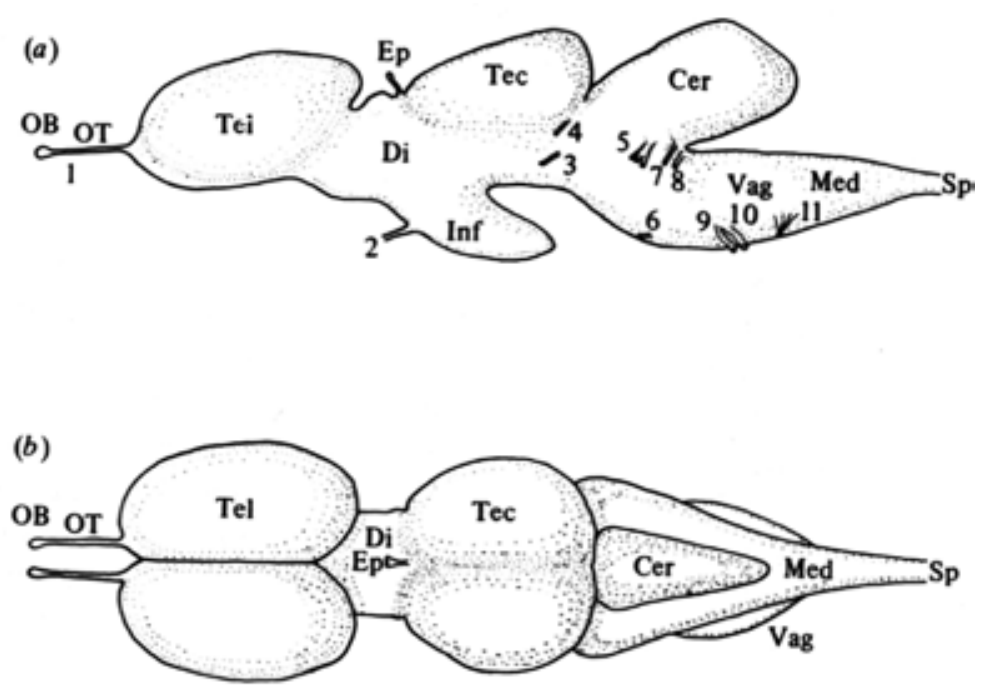

Lateral (a) and dorsal (b) views of a generalized lower vertebrate brain (e.g., teleost fish) to show the main superficial features. Cer, cerebellum; Di, diencephalon; Ep, epiphysis (pineal); Inf, inferior lobe of hypothalamus; Med, medulla oblongata; OB, olfactory bulb; OT, olfactory tract; Sp, spinal cord; Tec, optic tectum; Vag, vagal lobes; Tel, telencephalon; 1-11, cranial nerves (Adapted from Laming, 1981, p. 8). 
to present the general findings and theories regarding the development of the vertebrate forebrain and its evolutionary implications.

In early stages of development, the vertebrate forebrain has the shape of a vesicle, with dorsal and ventral walls that are thin and membranous. Laming (1981), by means of both ontogenetic and comparative anatomical evidence, presents an tentative overview of the telencephalon's evolution. Laming's proposed line of evolution is constituted in part by existing and extinct species; it begins with a hypothetical species that has a primitive non-differentiated telencephalon and evolves into three different "branches" ending in Teleosts, Amphibians, and Elasmobranchs.

In Amphibians and Elasmobranchs there is a pattern of evagination of the thick sidewalls of the telencephalic vesicle which causes both walls to meet in the median plane (forming two lateral ventricules) (Braford, 1995; Laming, 1981). Conversely, in holosteans and teleosts the same lateral walls of the telencephlalon evert.

Laming (1981) found that the differences in forebrain development and evolution suggested that the comparisons between forebrain functions in vertebrates rarely can be made on the basis of homology, and instead, analogy seemed to be a better approach. Nevertheless, more recent evidence that will be introduced here contradicts Laming's suggestion of using an "analogy approach" (e.g., Salas et al., 2006). Specifically the following sections will describe a research program which follows a more "conservative" approach in terms of forebrain structures and the associated and learning phenomena.

\section{Evolution of the brain and behavior in vertebrates: From fish to mammals}

Salas et al. (2006) contrast two important theories about the evolution of the brain and its functions from ancient vertebrates to mammals. The dominant "classical theories" of the early twentieth century proposed that brain evolution occurred over several successive stages, and consequently, the complexity of structures and functions have increased to what we can now see in the advanced cognitive capabilities of mammals. In terms of these theories, the fish telencephalon would consist mainly of a subpallium and a very small and primitive paleocortex, both entirely dedicated to sensory functions (particularly olfaction) and with relatively simple neural circuits. Moreover, given that more complex structures (e.g., caudate, putamen, hippocampus, and neocortex) were considered to appear later in more recent species, fish behavior was, therefore, assumed to be mainly reflexive or instinctive (Salas et al., 2006).

Savage's (1969) experiments constitute examples of the evidence that supported the aforementioned understanding of fish neurobehavioral functioning held during the early and mid-twentieth century. Savage reported that the failure in acquisition and retrieval of shock avoidance tasks in forebrainless fish was neither due to a reduced sensitivity to aversive stimulus (shock), nor a failure in a manifestation of signs of fear. Savage also reported that a) normal and forebrainless fish showed similar speeds in feeding, even when the levels of food deprivation and appetitive behavior were comparable; b) the removal of the forebrain did not interfere with the ability to learn simple simultaneous spatial discrimination; and c) the introduction of a five second delay between response and reward caused the extinction of discrimination in the telencephalon ablated fish, but not, however, in the non operated fish. Finally, Savage reported that the removal of the forebrain did not have an important effect on feeding rate.

As Salas et al. (2006) pointed out, because across different research programs the systematic removal of the telencephalon of fish did not show any impairment in sensory, motor, or motivational processes (as Savage's experiments clearly showed), the idea about the progressive evolution of the brain from fish to other more evolved vertebrates was maintained. Moreover, Laming (1981) defended a similar position when he stated that any functional or anatomic similarity between early vertebrates (e.g., fish) and more recent species would be better explained as analogies.

Nevertheless, at the end of the twentieth century researchers began to find unique ways to study 
the relationship between fish behavior and certain areas of the brain. The use of more precise techniques and experimental procedures revealed evidence that the forebrain in teleost fish is involved in emotional, social and, reproductive behavior, as well as in learning and memory (e.g., Flood \& Overmier, 1981; Overmier \& Hollis, 1983, 1990; Savage, 1980). In conjunction with this evidence, other neuroethological, comparative, developmental and neuroanatomical evidence lead to a different understanding of vertebrate brain evolution.

Instead of assuming a continuous and progressively linear complex evolution of the vertebrate brain, it seemed more plausible to assume that parallel radiations evolved independently from a remote common ancestor, from which vertebrates inherited some basic features of brain and behavior organization. Consequently, the increases in brain size and complexity occurred in different periods and in many members of the vertebrate family, including fish (Laming, 1980; Salas et al., 2003). As shown in Figure 2, the brain of extant and hypothetical vertebrates, although displaying noticeable morphological differences, can be understood as a combination of both primitive and derived characteristics. Following Salas et al. (2006), the-

\section{FIGURE 2}

Three hypothetical lines of telencephalon evolution

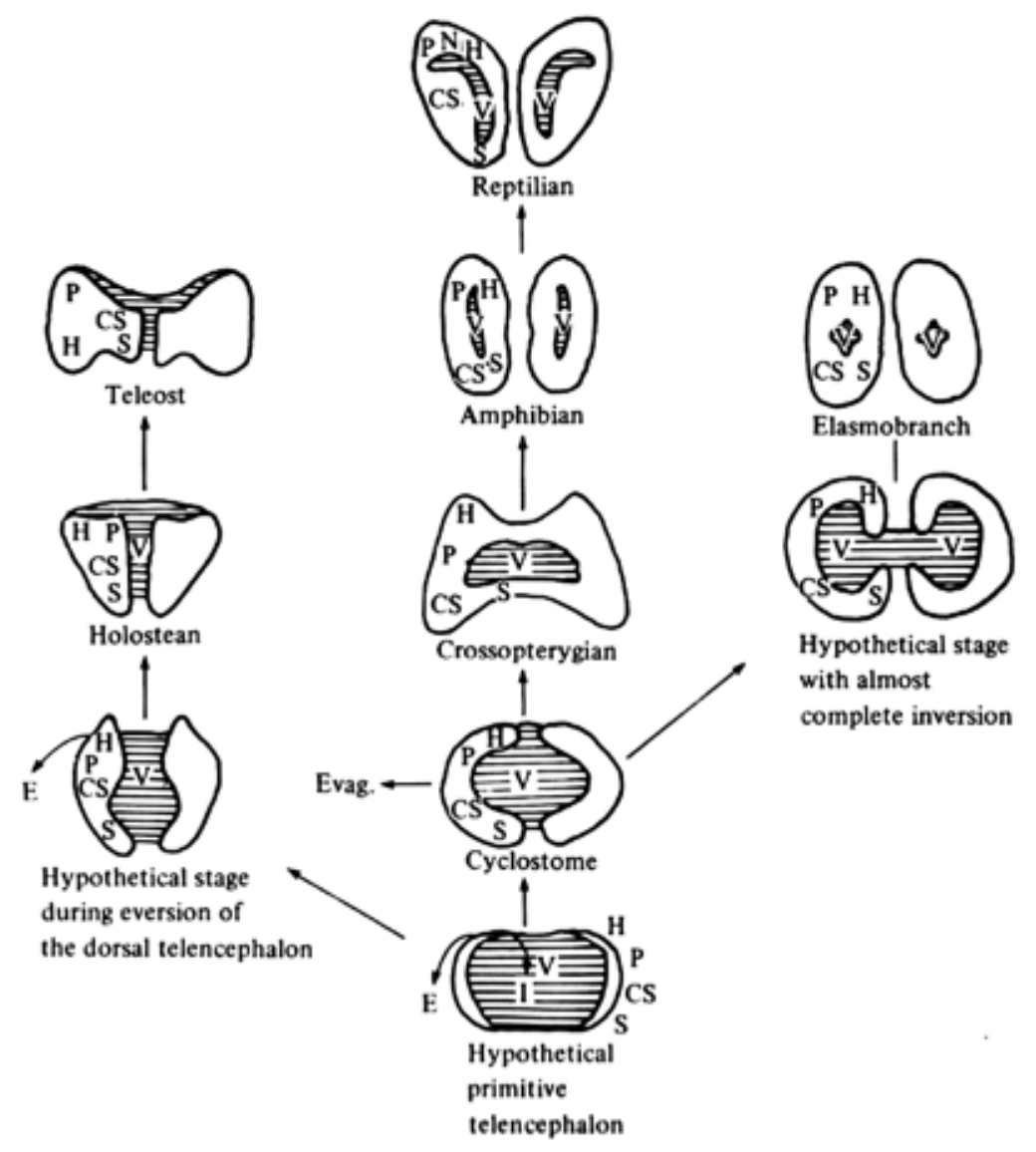

The graphic shows transversal brain sections of present, extant and hypothetical vertebrates which, although showing noticeable morphological differences, can be understood as a mixture of both primitive and derived characteristics.

$\mathrm{E}=$ Eversion; Evag. = Evagination; $\mathrm{I}=$ Inversion; $\mathrm{CS}=$ corpus striatum; $\mathrm{N}=$ Neocortex; $\mathrm{P}=$ Pyriform; $\mathrm{S}=\mathrm{Septum} ; \mathrm{H}=\mathrm{Hip}-$ pocampus; $\mathrm{V}=$ lateral ventricule.

Adapted from Laming (1981, p. 12). 
se morphological variations "can be conceived as variations of a common vertebrate plan” (p. 158), and it seems that the evidence today supports a more conservative understanding.

Whereas Laming (1981), Flood and Overmier (1981), Davis and Northcutt (1983), and Overmier and Hollis $(1983,1990)$ constitute the most complete reviews about the brain structures associated with different behavioral phenomena in teleost fish until the end of the twentieth century, more recent reviews are found in Portavella, Vargas, Torres, and Salas (2002) and Salas et al. (2006). The following section of the paper presents a general review, and if available, an update of the findings regarding the brain structures associated with memory, learning, and emotional behavior in fish. A special emphasis on emotional phenomena is made primarily because there is more available evidence coming from related research programs, and because some methodological alternatives derived from Hineline's (1977) parametric analysis of negative reinforcement and avoidance in species other than fish may lead to future comparative analysis and systematic replications.

\section{Non-associative learning}

Habituation is the reduction of responding as a consequence of repeated presentations or a prolonged exposure to a stimulus. Peeke, Peeke, and Willinston (1972) reported that after complete ablation of goldfish telencephalon, long-term habituation impairment was observed. Moreover, similar effects are reported by Laming and Ennis (1982), who explored the habituation of fright and arousal responses of goldfish and roaches. In Laming and Ennis' report, fright response appeared first and showed habituation in few trials, arousal response, however, appeared later but also habituated. The authors concluded that telencephalon ablation severely impaired habituation of arousal, though not fright responses. Furthermore that similar results (i.e. long-term impairment) have been found with telencephalon ablated Bettasplendens (Marino-Neto \& Sabatino, 1983).
In the case of sensitization, which refers to the increase in responding that results from the repeated presentations of a stimulus, and which is not attributable to peripheral processes, there was no report of learning impairments in fishwith telencephalic ablation (Overmier \& Curnow, 1969; Overmier \& Hollis, 1990).

\section{Associative learning}

Pavlovian conditioning is generally understood as the repeated pairing of a "neutral" stimulus and a hedonically powerful stimulus in such an arrangement that the neutral stimulus (CS) predicts the hedonic one (US). As a consequence of these pairings, the $\mathrm{CS}$ acquires behavior-controlling properties or Conditioned Response (CR) (Overmier \& Hollis, 1983, 1990).

Following the reviews of Overmier and Hollis $(1983,1990)$ and Salas et al. (2006), every experiment that tested the effects of telencephalic ablation on Pavlovian conditioning did not find any impairment of the learning process. This is a very general finding across a wide variety of procedures, associative indices, and range of delays (Overmier \& Hollis, 1983, 1990; Salas et al., 2006). In fact, Overmier and Hollis (1990) reported that telencephalon-ablated fish, exposed to high complexity classical conditioning processes, did not display impairment in their performance. Nonetheless, it is of special relevance that practically all the experiments on classical conditioning and its associated brain functions in fish have utilized electric shock or the presentation of a bright light as aversive stimulus(US). The relevance of this variable (i.e. type of aversive stimulation) may be the starting point for a research program that has not yet been developed.

Contrasting the lack of effects of telencephalic ablations, research conducted by Gómez, Durán, Salas, and Rodríguez (2010), Yoshida and Hirano (2010), and Rodríguez et al. (2005), exemplifies cumulated evidence supporting the notion that teleost's cerebellum is essential for the classical conditioning of several types of behavior (in the same way that it is for mammals). Rodriguez et 
al. (2005), and Gomez et al. (2010) analyzed the involvement of the cerebellum in the classical conditioning of motor and emotional responses and on spatial cognition. The authors reported that a) cerebellum lesions in goldfish impair the classical conditioning of a simple eye-retraction response, a phenomenon analogous to the eye blink conditioning described in mammals; b) autonomic emotional responses (e.g., heart rate classical conditioning) were also impaired by cerebellum lesions and; c) goldfish with cerebellum lesions presented a severe impairment in spatial cognition. Lastly, the authors reported that the observation of normal swimming activity or obstacle avoidance indicated that cerebellum lesions did not produce any observable motor deficit, and the lesions did not interfere with the occurrence of unconditioned motor or emotional responses. Rodriguez et al. (2005), Yoshida and Hirano (2010), and Gómez et al. (2010) reached the same conclusion; the functional involvement of the teleost's cerebellum in learning and memory is very similar to that of mammals. Subsequently, the authors suggest that the cognitive and emotional functions of the cerebellum may have evolved early in vertebrate evolution, having been conserved along the phylogenetic history of the extant vertebrate groups.

\section{Spatial learning}

Spatial cognition, sometimes considered as a form of relational memory, has been repeatedly associated with the function of the hippocampal formation in mammals (Portavella \& Vargas, 2005). Recent evidence shows that at least some learning and memory capabilities of teleost fish are as complex as those of mammals and birds and that they are likely based on homologous neural mechanisms (Portavella \& Vargas, 2005; Salas et al. 2006; Wullimann \& Mueller, 2004). Two areas of research related to the neurological mechanisms of spatial learning in fish will be reviewed here, successive reversal learning of spatial tasks and the role of pallial areas on the spatial behavior of fish.

Successive reversal learning of spatial tasks. The first comparative reports related with fish spatial behavior replicated the same finding obtained with other forms of behavior (e.g., discrimination); that is, in contrast with mammals, fish do not seem to improve their performance when they are required to repeatedly and successively reverse the previous trained response (Overmier \& Hollis, 1990). In sum, successive reversal learning is the improvement some animals display in their facility for mastering consecutive alternating problems and "attain a level where new problems are mastered with minimal errors” (Overmier \& Hollis, 1983, p. 279). An example of such a procedure would be teaching an animal an instrumental discrimination, then having it learn the opposite, and subsequently reversing the learning conditions again. Both the process and outcome are called successive reversal learning and under specific conditions, animals improve in the mastering of reversals (Overmier \& Hollis, 1983; Warren, 1961).

Following Overmier and Hollis' (1990) review of Frank, Flood, and Overmier (1972) and Warren's (1961) experiments, the general conclusion is that telencephalic ablated fish performed poorly in a spatial, successive reversal task compared to both normal fish or subjects with their olfactory bulbs removed. These early findings confirm the role of the telencephalon in spatial behavior of fish, but they also relate to the general debate about the existence of successive reversal learning in fish.

Overmier and Hollis (1990) reported that fish without brain lesions "[did not] improve when they were repeatedly required to reverse successfully the previous trained response" (p. 217), and the telencephalon ablated fish were different only in the fact that, compared to the "normal" fish which did not reduce errors across reversals, they actually showed lower performance across successive reversals, Interestingly, the subsequent findings of López, Bingman, Rodríguez, Gómez, and Salas (2000) seem to contradict the conclusions of Overmier and Hollis (1990). Lopez et al. found that goldfish with bilateral telencephalic ablation, sham operated or intact, were successfully trained in a spatial constancy task or in a directly cued task. The results showed that telencephalic ablation selectively impaired reversal learning in the animals trained in 
the spatial constancy procedure; specifically, ablated animals in this procedure reversed more slowly than control animals. In contrast, telencephalic ablation did not produce any interfering deficit during reversal in the animals trained in the cued task. Apart from providing additional evidence that the fish telencephalon is crucial in the spatial representations of fish -in a similar way that the hippocampus is central for the spatial representations of other vertebrates-, the findings of López et al. (2000) also relate to the issue of whether or not fish are able to display successive reversal learning. Even though the experiments of Lopez et al. (2000) were not designed to answer this matter, the fact that goldfish showed successful reversal in at least one occasion may imply that different procedures might produce successive reversal learning in fish.

The role of pallial areas in the spatial behavior of fish. Portavella and Vargas (2005) identified the lack of evidence regarding whether or not pallial areas of the fish brain were distinctively involved in different learning processes. To address this issue, they examined the effect of selective ablation of the medial and lateral pallium (MP and LP, respectively) on both the two-way avoidance and reversal spatial learning in goldfish. Even though the authors concluded that MP lesions selectively impaired the acquisition of the avoidance task (which will be discussed in more detail in the next section), LP ablations impaired the spatial task without affecting the the avoidance performance of fish. For Portavella and Vargas (2005) these results indicated that the MP and LP are functionally different and necessary for emotional and spatial learning in fish.

At a more conceptual level, the previous data supports the general hypothesis introduced at the beginning of this paper: "a sketch of [some] regions of the limbic system, and their associated functions, were present in the common ancestor of fish and terrestrial vertebrates 400 million years ago" (Portavella \& Vargas, 2005, p. 2800). For example, LP and $\mathrm{MP}$ of ray finned fish (i.e. actinopterygian) seem to be the most likely homologues of the hippocampus and amygdala of mammals (Salas et al. 2006). Finally, additional relevant evidence of homologies between fish and other vertebrates can be found in a research program that is more than 10 years old (e.g., Gómez, Durán, Salas, \& Rodríguez, 2010; Collins \& Waldeck, 2006; Rodríguez, et al., 2005; Broglio et al., 2005; Salas, Broglio, \& Rodríguez, 2003; Broglio, Rodríguez \& Salas, 2003; López, Vargas, Gómez \& Salas, 2003; López, Gómez, Vargas, \& Salas, 2003; Rodríguez, López, Vargas, Gómez, Broglio \& Salas, 2002; Durán, Vargas, Salas, \& Papini, 2000; López et al., 2000; Salas, Rodríguez, Vargas, Durán \& Torres, 1996; Salas, Broglio, Rodríguez, López, Portavella \& Torres, 1996; Rodriguez, Duran, Vargas, Torres \& Salas, 1994; Salas et al., 2006). For a recent review of this research program please refer to Salas et al. (2006).

\section{Emotional learning: Avoidance}

In a typical avoidance task, events are arranged so that a few seconds following the presentation of a brief stimulus (warning stimulus, sometimes called Conditioned Stimulus - CS) a second stimulus, typically delivered in the form of an electric shock, is presented. This stimulus is traditionally called aversive stimulus or Unconditioned Stimulus (US) . These contingent presentations of the CS and US are scheduled to happen unless the individual makes a designated response, in which case the aversive stimulus (US) is omitted (Overmier \& Hollis, 1990). In early trials, the animal responds in order to "terminate" the US (i.e. escape behavior). After experiencing some trials of this "escape form", the animal may begin to respond to the "warning stimulus" (CS), in other words, the animal begins to instrumentally prevent the aversive stimulus.

As Overmier and Hollis (1990) described, "the conundrum of avoidance learning is how the omission of an event can function to reinforce avoidance behavior; after all the animal makes the designated response and nothing happens" (p. 215). The cognitive approach to this critical issue suggests that the individuals exposed to this type of procedures develop expectations about the aversive event to occur, and consequently, a hypothetical internal state of "fear" would be 
reduced once the instrumental response is performed. What is more, the reduction of the fear state constitutes the explanation for the responding increase and maintenance. Even though several attempts to solve this issue have been undertaken (e.g., Hineline, 1984), the discussion remains open. One of the alternatives to clarify this issue has been the analysis offered by Hineline (1977)", which rather than being grounded in a conceptual level, is a behavioral approach heavily empirically and procedurally oriented. Hineline's analysis will be briefly described here because apart from offering a different approach for the interpretation of the aversive control phenomena, it may suggest a set of possible experimental preparations and research questions that could be explored in future neuroethological fish research.

The previously described basic avoidance procedure has been reviewed by Hineline (1977) in his exhaustive paper about avoidance and negative reinforcement phenomena and procedures. Hineline offered a different understanding of the continuity between escape and avoidance procedures by proposing a list of components that could be manipulated in any negative reinforcement preparation. One of the main contributions of Hineline's components analysis is that it suggests potential experimental procedures that may generate and maintain negatively reinforced behavior (i.e. escape or avoidance behavior), but still would be different from the standard preparation described at the beginning of this section. Furthermore, Hineline's ideas are presented here because of the long-lasting conceptual argument regarding the role of Pavlovian and instrumental contingencies to explain avoidance and escape behavior. Although Hineline's ideas do not completely resolve the debate, they do reduce some ambiguity by suggesting that there may be other procedural alternatives that do not necessarily fit in the typical understanding of these phenomena and might lead to different sources of evidence. In brief, Hineline may be arguing that the discussion could be based on a pseudo-problem.

The parameters Hineline (1977) described are the following: a) a continuous or non-continuous aversive stimulation presentation (which can be manipulated in timing, frequency, probability, etc.); b) an opportunity for responses to occur and be counted or measured (e.g., in most of the procedures the operandum is removed or a door closes and the first response the subject presents terminates the trial); and c) an occasion when the specified response can affect the occurrence of

Figure 3

Components of three different negative reinforcement procedures

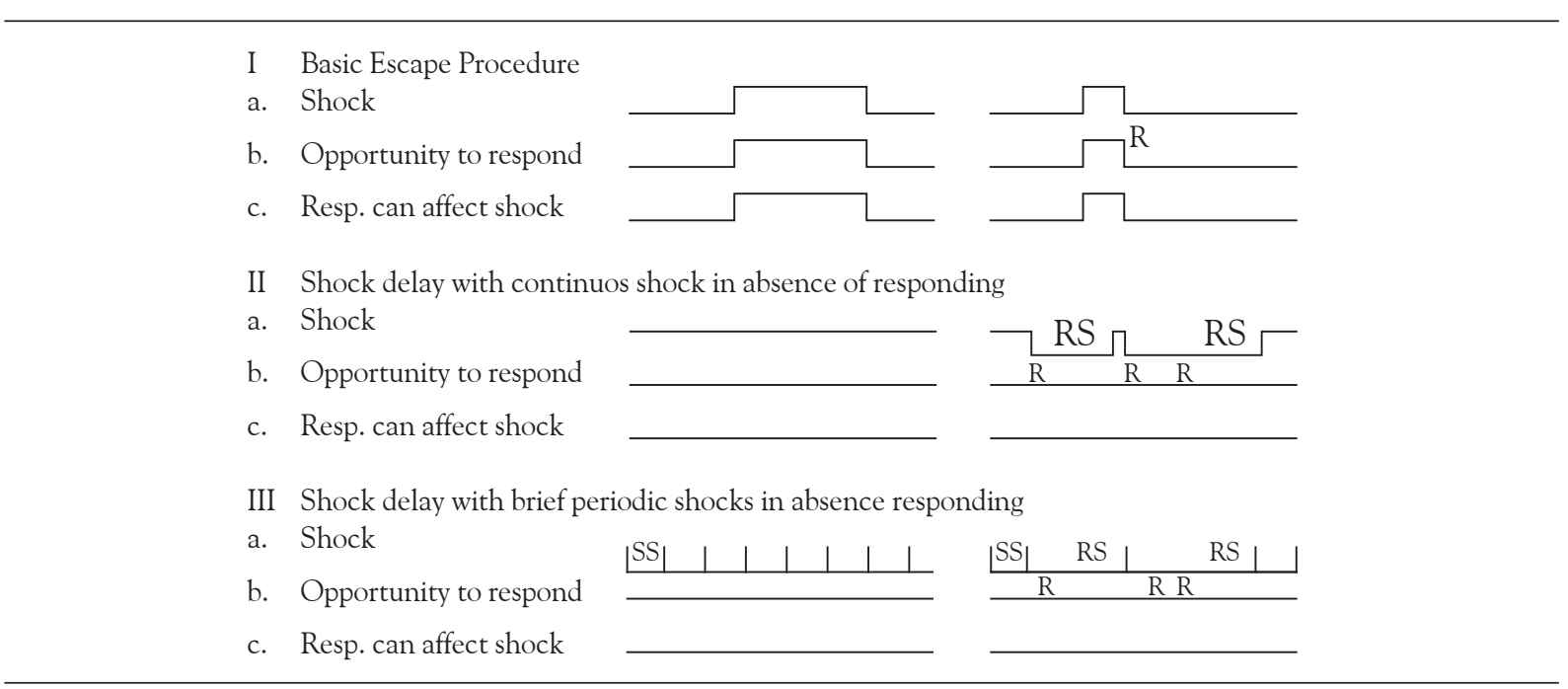

Adapted from Hineline (1977). 
the aversive stimulation (which can vary in terms of being present continuously, being intermittent, or having different timings). Figure 3 constitutes a representation of these parameters and different procedures.

The components of three different negative reinforcement procedures that allow for the comparison of escape preparations to other kind of aversive control arrangements are shown in Figure 3. Electric shock exemplifies the aversive stimulus because it is the most common form of stimulation used in this kind of experiments. Time is indicated from left to right and " $R$ " represents the moment when responses may occur. Part I constitutes the basic escape procedure, where (a) represents the aversive stimulus presentation (e.g., shock), (b) the opportunity the organism has to respond (R), and (c) the moment when the response can affect the presentation of the aversive stimulus (i.e. deletion or delay). This is a typical discrete trial procedure because (a), (b) and (c) are not constantly in force. Conversely, Part II represents a situation where the aversive stimulus (a), the opportunity to respond (b), and the effect of responding on the presentation of the aversive stimulus are continuously valid. Also, on the next onset of the aversive stimulus a "delay" is introduced which is proportional to the number of responses the organism shows (Response-Shock interval - RS). Part III represents a more complex procedure in which the aversive stimulus (a) is presented briefly and periodically. The organism has the opportunity to respond (b) at any time and the contingency specifies that the interval between shocks (Shock-Shock interval - SS) is half the time of the Response-Shock interval (RS). In other words, every time the organism responds, the time that will elapse between the response and the next shock is twice the amount of time scheduled for the SS period (adapted from Hineline, 1977, p. 365).

Hineline (1977) based his analysis of the previously described components on a review of several experimental findings in negative reinforcement, escape and avoidance. He considered albeit many of the procedures used in behavioral research followed the typical arrangement of having a warning stimulus and a complete delay of the aversive stimulus contingent on the designated response, alternative and less popular experiments have also shown the acquisition and maintenance of negative reinforced behavior. Moreover, Hineline pointed out that these negatively reinforced behaviors manifested under circumstances in which the subjects (a) reduced, but not fully avoided, the contact with aversive stimuli by means of their responding (e.g., under a schedule of reduction of density of the shock the subject still occasionally experiences the aversive stimuli); and (b) the subjects responded during the procedure in the absence of warning stimulus.

It is important to note that Hineline's (1977) review was based exclusively on experiments with rats and pigeons; however, systematic research of the same type with fish subjects has not been accomplished to date. The reader should, therefore, be aware that the following reviewed experiments are based exclusively on one of the possible configurations of negative reinforcement contingencies. Furthermore, the effects of other arrangements are still open for exploration, aside from the "conundrum" identified by Overmier and Hollis (1990).

Early research in aversive control of fish behavior was focused on confirming learning acquisition of normal subjects (non-ablated) after being exposed to several variations of the typical avoidance procedure (using a warning stimulus and a complete deletion of the aversive stimulation as a consequence of the subject's response) (Overmier \& Hollis, 1983, 1990). A second generation of experiments, however, was focused on the consequences of forebrain removal for emotional learning, while also exploring possible differences in acquisition and/or long term performance (i.e. once the task is mastered) due to brain damage. Finally, the current research trend focuses on identifying specific areas of the fish telencephalon which may underlay the emotional behavioral processes. The experimental findings related to these two research generations will be reviewed in the following section.

Consequences of forebrain removal in avoidance learning. Hainsworth, Overmier, and Snowdon (1967) conducted one of the first studies focused 
on the effects of telecenphalic ablation during the acquisition of an avoidance task in fish. The authors tested normal, sham operated, or forebrain ablated goldfish in the acquisition, extinction, and retention of an instrumental avoidance response with either a short or a long delay between operations and testing. Hainsworth et al. (1967) found that forebrain removal severely impaired or prevented acquisition, greatly reduced resistance to extinction, and completely abolished a previously learned response. In addition, the authors reported that the average escape latencies did not differ between groups, indicating that the deficit was specific to avoidance learning. Giving that similar findings were reported by Overmier and Hollis $(1983,1990)$, the general conclusion can be that the learning of the avoidance response is not completely blocked when ablation has occurred; instead, the response takes more time to develop, is unstable, and extinguishes very rapidly.

Effects of brain damage over long term performance. Regarding the effects of forebrain ablation, once the avoidance response is developed, the main reported outcome is a complete elimination of the avoidance response (Hainsworth et al., 1967). Moreover, attempts to retrain the subjects that were exposed to the ablation procedure, revealed little, if any, improvement. These findings regarding acquisition and long term performance lead to three conclusions: first, they suggested the existence of different fish brain structures specialized for acquisition and maintenance of the avoidance response. Second, these results also demonstrate a clear dissociation between the brain structures that underlay classical conditioning and avoidance learning in fish. Finally, these findings suggest that "avoidance behavior is dependent on brain mechanisms that are different from those of simple pavlovian and instrumental learning" (Overmier and Hollis, 1990, p. 216).

Specific telencephalic areas and functions underlying emotional behavior. Contemporary research has been focused on the isolation of the specific areas of the fish telencephalon that may underlay emotional/behavioral processes. This research trend can be divided in two main areas: a) stimula- tion or ablation of the Medium and Lateral Pallium (MP and LP), and b) chemical stimulation of the brain. Research on the stimulation or ablation of the MP and LP has provided the basis for the same conclusion that certain pallium areas of the fish brain have a structural and functional continuity with specific brain areas in mammals such as the amygdala and hippocampus (Portavella \& Vargas, 2005). Furthermore, some researchers have concluded that the MP, like the amygdala, seems to underlie behaviors which involve emotional components. Whereas selective lesions of the MP affect and disorganize aggressive, reproductive, and parental behavior, focal electrical stimulation in the MP has been found to elicit arousal, defensive behavior, and escape responses (Salas et al., 2006).

Portavella et al. (2004) hypothesized that MP is responsible for the retention of a conditioned active avoidance response in goldfish. The authors were interested in the effects of the ablation of MP and LP on the retention of a conditioned avoidance response previously acquired in two experimental conditions. In the first experiment, goldfish were trained in non-trace avoidance conditioning; in the second experiment, fish were trained in trace avoidance conditioning in which temporal cues were crucial for the learning process. In the case of the MP lesion, deficits in the retention of the avoidance response were observed in both procedures (trace and non-trace). Conversely, LP lesions impaired the retention of the avoidance response only in the trace-conditioning procedure. The authors, therefore, confirmed the hypothesis that two diffe rent systems of memory exist in fish: an emotional memory system in which the MP is involved; and a spatial, relational, or temporal memory system in which the LP is involved. What is more important is that these effects were similar to those produced by amygdalar and hippocampal lesions in mammals (Portavella et al., 2004).

Evidence for additional dissociations comes from experiments in which goldfish were trained immediately after they were subject to MP lesions. These experiments showed that fish can only im prove in escape behavior, but not in avoidance 
behavior. In other words, escape and avoidance seem to be behavioral phenomena that depend on different brain structures. Additionally, the MP lesion impairment on avoidance memory is as severe as those produced by the ablation of the whole telencephalon, thereby indicating that the MP is a critical area for this behavioral function in teleost fish (Portavella, Torres, \& Salas, 2004; Portavella, Torres, Salas, \& Papini, 2004; Portavella, Vargas, Salas, \& Papini, 2003; Salas et al., 2006).

The selective involvement of the MP in avoidance learning is confirmed in the failure of MPlesioned goldfish that are trained to express avoidance responses using spaced-trial procedures (i.e. one trial per day). Portavella et al. (2003) considered that widely spaced training allow for the evaluation of the ability of a discriminative stimulus to control an avoidance response in the absence of stimulus carry-over effects from prior recent trials. Accordingly, after validating this procedure with goldfish, Portavella, Torres, Salas, and Papini (2004) conducted a series of subsequent experiments using the widely spaced trial procedure, and they found that control and LP-lesioned goldfish exhibited significantly faster avoidance learning than animals with MP lesions. Based on this evidence, Portavella et al. (2004) concluded that the MP, not the LP, may be responsible for the frequently mentioned deficits in avoidance learning that follow lesions of the entire telencephalon.

Alternatively, the second contemporary research trend utilizes chemical stimulation in order to isolate specific areas of the telencephalon that may underlay the emotional-behavioral processes in fish. The main objective is to explore the similarities of behavioral effects produced by certain chemicals when comparing mammals to fish. The conceptual and empirical background of this research program is based on the study of neurochemical processes that are correlated with the acquisition and maintenance of different learned responses, including emotional behavior. In general, several studies focused on synaptic transmission processes have revealed that the neurochemical steps of Long-Term potentiation (LTP), a physiological correlate of learning and memory, involve the activation of postsynaptic $\mathrm{N}$-methyl-D-aspartate (NMDA) receptors; the activation of the Nitric Oxide Synthase enzyme (NOS) that leads to the synthesis of Nitric Oxide (NO); and the activation of the soluble GuanylateCyclase (sGC) enzyme which increases the formation of cyclic Guanosine Monophosphate (cGMP) (Xu et al., 2009).

In mammals, it has been found that intraamygdaloid infusions of NMDA antagonists, such as aminophosphonopentanoic acid (AP5) and dizocilpine maleate (MK-801), prevent the acquisition of Pavlovian fear conditioning and avoidance conditioning. For example, Miserendino, Sananes, Melie, and Davis (1990) proposed that if the receptors for NMDA are to have a critical role in synaptic plasticity and NMDA antagonists (such as AP5) prevent several neural mechanisms that may underlie learning and memory, then the introduction of NMDA antagonists would interfere with memory formation in conditioning tasks. Consequently, after showing that NMDA antagonists infused into the amygdala blocked the acquisition, and not the expression of fear conditioning, Miserendino et al. (1990) concluded that an NMDA-dependent process in the amygdala may underlie associative fear conditioning. Similar studies have replicated this type of neurological phenomena (e.g., Maren, 2001).

Likely due to the previously hypothesized evolutive continuity, the same NMDA receptors found in mammals' brains are found to be densely concentrated in the goldfish telencephalon. Accordingly, it is not surprising that intracranial administration of NMDA receptor antagonists affects the acquisition of avoidance and fear conditioning in fish. What is more is that this effect seems to work in a dose-dependent manner (Salas et al., 2006).

In addition, related research has found coherent neurological dissociations between the acquisition of fear conditioning tasks and memory consolidation in goldfish. The insertion of D-AP5 in the goldfish telencephalon immediately following training was found to impair memory consolidation of avoidance conditioning. These findings lead to the hypothesis that NMDA receptor antagonists 
impaired learning by disrupting the neural mechanisms of acquisition and not, as previously thought, by blocking memory storage or retrieval processes (Xu, Bazner, Qi, Johnson \& Freidhoff, 2003; Xu, et al., 2009). Given that L-NAME is a Nitric Oxide -NO- inhibitor, and LY-83583 is a Cyclic Guanosine Monophosphate-cGMP-inhibitor, Xu et al. (2009) utilized these chemicals to explore the relationships between NMDA receptor antagonists and the neural processes of acquisition.

After confirming that L-NAME and LY-83583 produced significant anterograde and retrograde amnesia at doses that did not impair performance processes during avoidance conditioning, $\mathrm{Xu}$ et al. (2009) concluded that the NMDA receptors may be involved in learning or the process that is completed during training; conversely, the authors suggested that the NO and CGMP are involved in memory consolidation or the process that is completed sometime following the learning experience. Finally, alternative experiments have provided additional support for this hypothesis. For example, it has been found that NMDA receptors and protein kinases play an important functional role in LTP formation, not only in the telencephalon, but also in the optic tectum and brainstream of fish (Salas et al., 2006).

\section{Conclusions}

Specific brain areas and neurochemical functions that have been identified as underlying different learning phenomena in fish were described here. In doing so, I presented the neuroethological approach, a brief review of the teleost fish brain anatomy and ontogeny, and a discussion about the evolutionary relationships between fish and mammals. Contrary to the theory dominating most of the twentieth century, there is strong evidence of the existence of homologies in neurobehavioral functions between fish and mammals.

Although habituation has been understood to be affected by the removal of telencephalic areas, experimental attempts to demonstrate sensitization and classical conditioning impairments after removal of these areas have failed to produce learning deficits in fish. Even though an important number of studies have reported a general ineffectiveness of telencephalic ablations on classical conditioned responses across procedures, range of delays, indexes, etc., it was noted that electric shock has been utilized exclusively on this experimental preparations; consequently, future systematic replication using alternative forms of aversive stimulation are, therefore, merited.

Contrary to the effect of telencephalic ablations, cerebellum lesions have shown detrimental effects in classical conditioning of simple eyeretraction responses, autonomic responses, and spatial learning. As a result, increasing evidence supports the functional involvement of the teleost's cerebellum in learning and memory. The similarity of the cerebellum's role in teleost and mammals suggests that the functions of this brain structure may have evolved early in vertebrate evolution.

In regards to spatial learning, the evidence reviewed here supports the hypothesis that there are at least some spatial learning capabilities found in mammals and birds that can be also found in teleost fish. Furthermore, the evidence suggests that these learning capabilities are likely based on homologous neural mechanisms. Even though the general finding that fish without brain lesions lack improvement when they are repeatedly required to reverse a previous trained response, telencephalon ablated fish have displayed worse performances than the intact fish that have been exposed to identical reversal training. As such, the literature seems to favor the notion that telencephalic ablation affects spatial learning when successive reversal tasks are implemented. However, it was also noted that indirect evidence suggests fish may, indeed, be capable of presenting reversal learning but under different preparations (Lopez et al., 2000).

Whereas early research programs identified that forebrain removal selectively impaired avoidance response acquisition, reduced resistance to extinction, and abolished previously learned behavior, the recent availability of more sensitive and precise techniques have allowed researchers to identify the critical role of the Medium Pallium (MP) areas in fishes' emotional learning. Further- 
more, neurobehavioral functions of MP in fish appear to be similar to the functions of the amygdala in mammals.

In regards to the research methods in emotional learning, a critical approach based on Hineline's (1977) work was introduced in order to argue that research on avoidance and escape learning has been narrowly oriented to a particular set of parameters and procedures. As a result, it was suggested that new research possibilities may emerge from the manipulation of Hineline's parametric analysis.

Finally, the literature has clearly shown accumulating evidence of similar neurochemical processes that underlie the acquisition and maintenance of emotional behavior in fish and mammals. Intracranial administration of NMDA receptor antagonists affects, in a dose dependant manner, the acquisition of avoidance and fear conditioning in fish. On the other hand, NO and cGMP appear to be involved in the learning consolidation process that occurs, at some point, subsequent to the learning period.

\section{References}

Braford, M. R., Jr. (1995). Comparative aspects of forebrain organization in the ray-finned fishes: Touchstones or not? Brain, Behavior and Evolution, $46(4-5), 259-274$.

Broglio, C., Gómez, A., Durán, E., Ocaña, F. M., Jiménez-Moya, F., Rodríguez, F. et al. (2005). Hallmarks of a common forebrain vertebrate plan: Specialized pallial areas for spatial, temporal and emotional memory in actinopterygian fish. Brain Research Bulletin, 66 (4-6), 277-281.

Broglio, C., Rodríguez, F. \& Salas, C. (2003). Spatial cognition and its neural basis in teleost fishes. Fish and Fisheries, 4 (3), 247-255.

Collins, L. E. \& Waldeck, R. F. (2006). Telencephalic ablation results in decreased startle response in goldfish. Brain Research, 1111 (1), 162-165.

Davis, R. G. \& Northcutt, R. E. (Eds.). (1983). Fish Neurobiology. Ann Arbor: The University of Michigan Press.
Durán, E., Vargas, J. P., Salas, C. \& Papini, M. R. (2000). Effect of telencephalic ablation on appetitive instrumental learning in the goldfish (carassiusauratus). Psicothema, 12 (4), 520-524.

Flood, N. B. \& Overmier, J. B. (1981). Learning in teleost fish: Role of the telencephalon. In P. R. Laming (Ed), Brain mechanisms of behavior in lower vertebrates (pp. 259-279). Cambridge: Cambridge University.

Frank, A. H., Flood, N. C. \& Overmier, J. B. (1972). Reversal learning in forebrain ablated and olfactory tract sectioned teleost, carassiusauratus. PsychonomicScience, 26, 149-151.

Gómez, A., Durán, E., Salas, C. \& Rodríguez, F. (2010). Cerebellum lesion impairs eyeblink-like classical conditioning in goldfish. Neuroscience, 166 (1), 49-60.

Hainsworth, F. R., Overmier, J. B. \& Snowdon, C. T. (1967). Specific and permanent deficits in instrumental avoidance responding following forebrain ablation in The Goldfish. Journal of Comparative and Physiological Psychology, 63 (1), 111-116.

Hineline, P. N. (1977). Negative reinforcement and avoidance. In W. K. Honig \& J. E. R. Staddon (Eds.), Handbook of operant behavior (pp. 364-414). Englewood Cliffs, NJ: Prentice Hall.

Hineline, P. N. (1984). Aversive control: A separate domain? Journal of the Experimental Analysis of Behavior, 41 (3) 495-509.

Laming, P. R. (Ed.). (1981). Brain mechanisms of behavior in lower vertebrates. Cambridge: Cambridge University Press.

Laming, P. R. \& Ennis, P. (1982). Habituation of fright and arousal responses in the teleostscarassiusauratus and rutilusrutilus. Journal of Comparative and Physiological Psychology, 96 (3), 460-466.

López, J. C., Bingman, V. P., Rodríguez, F., Gómez, Y. \& Salas, C. (2000). Dissociation of place and cue learning by telencephalic ablation in goldfish. Behavioral Neuroscience, 114 (4), 687-699.

López, J. C., Broglio, C., Rodríguez, F., Thinus-Blanc, C. $\&$ Salas, C. (2000). Reversal learning deficit in a spatial task but not in a cued one after telencephalic ablation in goldfish. Behavioral Brain Research, 109 (1), 91-98. 
López, J. C., Gómez, Y., Vargas, J. P. \& Salas, C. (2003). Spatial reversal learning deficit after medial cortex lesion in turtles. Neuroscience Letters, 341 (3), 197-200.

López, J. C., Vargas, J. P., Gómez, Y. \& Salas, C. (2003). Spatial and non-spatial learning in turtles: The role of medial cortex. Behavioral Brain Research, 143 (2), 109-120.

Maren, S. (2001). Neurobiology of pavlovian fear conditioning. Annual Review Neuroscience, 24, 87-931.

Marino-Neto, J. \& Sabbatini, R. M. (1983).Discrete telencephalic lesions accelerate the habituation rate of behavioral arousal responses in siamese fighting fish (bettasplendens). Brazilian Journal of Medical and Biological Research, 16 (3), 271-278.

Miserendino, M. J. D., Sananes, C. B., Melie, K. R. \& Davis, M. (1990). Blocking of acquisition but not expression of conditioned fear-potentiation startle by NMDA antagonists in the amygdala. Nature, 345 (6277), 716-718.

Overmier, J. B. \& Curnow, P. F. (1969). Classical conditioning, pseudoconditioning, and sensitization in "normal" and forebrainless goldfish. Journal of Comparative and Physiological Psychology, 68 (2 PART 1), 193-198.

Overmier, J. B. \& Hollis, K. L. (1983). The teleostean telencephalon in learning. In R. G. Northcut \& R. E. Davis (Eds.), Fish Neurobiology (pp. 265-284). Ann Arbor: The University of Michigan Press.

Overmier, J. B. \& Hollis, K. L. (1990). Fish in the think tank: Learning, memory and integrated behavior. In R. P. Kesner \& D. S. Olton (Eds.), Neurobiology of comparative cognition (pp. 204-236). Hillsdale: Lawrence Erlbaum Associates.

Portavella, M., Salas, C., Vargas, J. P. \& Papini, M. R. (2003). Involvement of the telencephalon in spaced-trial avoidance learning in the goldfish (carassiusauratus). Physiology and Behavior, 80 (1), 49-56.

Portavella, M., Torres, B. \& Salas, C. (2004). Avoidance response in goldfish: Emotional and temporal involvement of medial and lateral telencephalicpallium. Journal of Neuroscience, 24 (9), 2335-2342.

Portavella, M., Torres, B., Salas, C. \& Papini, M. R. (2004). Lesions of the medial pallium, but not of the lateral pallium, disrupt spaced-trial avoidance learning in goldfish (carassiusauratus). Neuroscience Letters, 362 (2), 75-78.

Portavella, M. \& Vargas, J. P. (2005). Emotional and spatial learning in goldfish is dependent on different telencephalicpallial systems. European Journal of Neuroscience, 21 (10), 2800-2806.

Portavella, M., Vargas, J. P., Torres, B. \& Salas, C. (2002). The effects of telencephalicpallial lesions on spatial, temporal, and emotional learning in goldfish. Brain Research Bulletin, 57 (3, 4), 397-399.

Peeke, H. V. S., Peeke, S. C. \& Williston, J. S. (1972). Long-term memory deficits for habituation of predatory behavior in the forebrain ablated goldfish (carassiusauratus). Experimental Neurology, 36 (2), 288-294.

Rodríguez, F., Durán, E., Gómez, A., Ocaña, F. M., Álvarez, E., Jiménez-Moya, F. et al. (2005). Cognitive and emotional functions of the teleost fish cerebellum. Brain Research Bulletin, 66 (4-6), 365-370.

Rodriguez, F., Durán, E., Vargas, J. P., Torres, B. \& Salas, C. (1994). Performance of goldfish trained in allocentric and egocentric maze procedures suggests the presence of a cognitive mapping system in fishes. Animal Learning and Behavior, 22 (4), 409-420.

Rodríguez, F., López, J. C., Vargas, J. P., Broglio, C., Gómez, Y. \& Salas, C. (2002). Spatial memory and hippocampal pallium through vertebrate evolution: Insights from reptiles and teleost fish. Brain Research Bulletin, 57 (3-4), 499-503.

Rodríguez, F., López, J. C., Vargas, J. P., Gómez, Y., Broglio, C. \& Salas, C. (2002). Conservation of spatial memory function in the pallial forebrain of reptiles and ray-finned fishes.Journal of Neuroscience, 22 (7), 2894-2903.

Salas, C., Broglio, C., Durán, E., Gómez, A., Ocaña, F. M., Jiménez-Moya, F. et al. (2006). Neuropsychology of Learning and Memory in Teleost Fish. Zebrafish, 3 (2), 157-171.

Salas, C., Broglio, C. \& Rodríguez, F. (2003). Evolution of forebrain and spatial cognition in vertebrates: Conservation across diversity. Brain, Behavior and Evolution, 62 (2), 72-82.

Salas, C., Broglio, C., Rodríguez, F., López, J. C., Portave1la, M. \& Torres, B. (1996). Telencephalic ablation 
in goldfish impairs performance in a 'spatial constancy' problem but not in a cued one. Behavioral Brain Research, 79 (1-2), 193-200.

Salas, C., Rodríguez, F., Vargas, J. P., Durán, E. \& Torres, B. (1996). Spatial learning and memory deficits after telencephalic ablation in goldfish trained in place and turn maze procedures.Behavioral Neuroscience, 110 (5), 965-980.

Savage, G. E. (1969). Some preliminary observations on the role of the telencephalon in food-reinforced behavior in the goldfish, Carassiusauratus. Animal Behavior, 17 (4), 760-772.

Savage, G. E. (1980). The fish telencephalon and its relation to learning. In S. O. E. Ebbesson (Ed.), Comparative Neurology of the Telencephalon (pp. 129-174). New York: Plenum.

Warren, J. M. (1961). The effect of telencephalic injuries on learning by paradise fish, macropodusopercularis. Journal of Comparative and Physiological Psychology, 54 (2), 130-132.
Wullimann, M. F. \& Mueller, T. (2004). Teleostean and mammalian forebrains contrasted: Evidence from genes to behavior. Journal of Comparative Neurology, 475 (2), 143-162.

Xu, X., Bazner, J., Qi, M., Johnson, E. \& Freidhoff, R. (2003). The role of telencephalic NMDA receptors in avoidance learning in goldfish (carassiusauratus). Behavioral Neuroscience, 117 (3), 548-554.

Xu, X., Bentley, J., Miller, T., Zmolek, K., Kovaleinen, T., Goodman, E. et al. (2009). The role of telencephalic $\mathrm{NO}$ and cGMP in avoidance conditioning in goldfish (carassiusauratus). Behavioral Neuroscience, 123 (3), 614-623.

Yoshida, M. \& Hirano, R. (2010). Effects of local anesthesia of the cerebellum on classical fear conditioning in goldfish.Behavioral and Brain Functions, 6, 20. Retrieved from http://www.behavioralandbrainfunctions.com/content/6/1/20 